\title{
Tecnura
}

\section{Diagnóstico tecnológico del uso de dispositivos programables en la industria boyacense. Caso de estudio: cadena agroindustrial de la panela}

\author{
Technological diagnosis of the use of programmable devices \\ in the boyaca industry: Case study - panela's agroindustrial chain
}

\author{
Ilber Adonayt Ruge Ruge1, Wilson Javier Pérez Holguín²
}

Fecha de recepción: 12 de agosto de 2016

Fecha de aceptación: 15 de febrero de 2017

Cómo citar: Ruge R., I.A. y Pérez H., W.J. (2017). Diagnóstico tecnológico del uso de dispositivos programables en la industria boyacense. Caso de estudio: cadena agroindustrial de la panela. Revista Tecnura, 21(52), 130-147. doi: 10.14483/udistrital.jour.tecnura.2017.2.a10

\section{Resumen}

Contexto: En este artículo se describen los resultados de un proceso de diagnóstico tecnológico tendiente a identificar el nivel de incorporación de los dispositivos programables (microcontroladores) y de los dispositivos de lógica programable (CPLD y FPGA) en los procesos productivos del sector agroindustrial de la panela, en el departamento de Boyacá. Cabe resaltar que el sector agroindustrial es uno de los que más aportan al producto interno bruto del departamento y es uno de los más priorizados en términos del potencial de negocios y entorno económico favorable de acuerdo con el Plan Estratégico Departamental de Ciencia, Tecnología e Innovación del Departamento de Boyacá.

Método: La metodología empleada consistió en obtener un inventario tecnológico (máquinas, equipos e infraestructura informática) e identificar los activos tecnológicos de las empresas relacionadas.

Resultados: Para la fase de recolección de información se visitaron cuatro empresas productoras de panela ubicadas en la hoya del río Suarez, de las cuales se encontró que solo una tienetienen un alto grado de automatización en sus procesos de producción, mientras que las otras presentan uno muy bajo o ninguno. La revisión de literatura evidenció que existen varias publicaciones en revistas indexadas en las que se describen importantes aportes al desarrollo del sector agroindustrial de la panela, aunque ninguno es realizado por universidades del departamento. En la etapa de análisis se identificaron las debilidades y oportunidades de las empresas del sector panelero en Boyacá.

Conclusiones: Todo lo anterior permitió definir un conjunto de propuestas de incorporación de las tecnologías programables para el desarrollo de proyectos de investigación, encaminados a cubrir las necesidades reales del sector panelero del departamento de Boyacá, tanto en el mejoramiento de los procesos de producción como en el de las condiciones de trabajo de las personas dedicadas a estas labores.

Palabras clave: diagnóstico tecnológico, dispositivos programables, dispositivos de lógica programable, producción de panela.

1 Ingeniero electrónico, magíster en Ingeniería de Control Industrial. Docente asistente de la Universidad Pedagógica y Tecnológica de Colombia, grupo de investigación I²E. Bogotá Tunja, Colombia. Contacto: ilber.ruge@uptc.edu.co

2 Ingeniero electrónico, magíster en Automatización, doctorado en Ingeniería. Docente asociado de la Universidad Pedagógica y Tecnológica de Colombia, Grupo GIRA-UPTC. Bogotá Sogamoso, Colombia. Contacto: wilson.perez@uptc.edu.co 


\section{Abstract}

Context: This paper describes the results of a technological diagnostic process aimed to identify the level of incorporation of programmable devices (e.g. microcontrollers) and programmable logic devices (e.g. CPLDs and FPGAs) in the productive processes of the panela agro-industrial sector in the Department of Boyacá. It should be noted that the agro-industrial sector is one of the largest contributors to the gross domestic product of the department and is one of the most prioritized in terms of business potential and favorable economic environment according to the Departmental Strategic Plan for Science, Technology and Innovation of the Department of Boyacá.

Method: The methodology used consists on to obtain a technological inventory (machines, equipment and IT infrastructure) and identify the technological assets of the related companies.

Results: During the information collection phase, four panela producer companies were visited, all of them located in the Hoya del Rio Suarez region, of which it was found that only one presents a high degree of automation of its production processes, while the others have a very low or no automation. The literature review evidenced that there are several publications in indexed journals that describe important contributions on the development of the agro-industrial sector of panela, although none of them are made by universities of the department. In the analysis stage, the weaknesses and opportunities of the companies dedicated to producing panela in Boyacá were identified. Conclusions: All of the above allowed defining a set of proposals for the incorporation of programmable technologies in the development of research projects aimed at meeting the real needs of the companies producing panela in the Department of Boyacá, both in the improvement of production processes as in the working conditions of the persons devoted to these tasks.

Keywords: technological diagnostic, programable devices, programable logic devices, panela producers.

\section{INTRODUCCIÓN}

La agroindustria se proyecta como un reglón importante para el desarrollo socio-económico del Departamento de Boyacá, dado el fuerte crecimiento de la demanda de alimentos frescos, semiprocesados y procesados, tanto en la región como en el país. Los países de América Latina presentan grandes diferencias tecnológicas con los países desarrollados, hecho que se evidencia en la capacidad de los primeros para absorber, imitar, adaptar e innovar tecnologías que puedan ser empleadas en sus diferentes procesos productivos, teniendo como punto de referencia las mejores prácticas internacionales de adaptación a la demanda y su correspondiente cambio tecnológico. De esta manera, es fundamental conocer el estado tecnológico de las diferentes industrias de la región para facilitar el planteamiento de proyectos de investigación que faciliten el mejoramiento de los procesos productivos, y aprovechar las oportunidades que brindan los planes de desarrollo departamental y las políticas de fortalecimiento en ciencia, tecnología e innovación, tanto del departamento como de la nación.

Este artículo presenta los resultados de un diagnóstico orientado a identificar el tipo de tecnología empleada en las pequeñas y medianas empresas relacionadas con la industria panelera del departamento de Boyacá. Como consecuencia, se elabora una propuesta de desarrollo tecnológico en las áreas de control, automatización y sistemas de información, basada en el uso de dispositivos programables (como microcontroladores) o de lógica programable (como los dispositivos de lógica programable compleja, o CPLD, por sus siglas en inglés, y los arreglos de compuertas programables en campo, o FPGA, por sus siglas en inglés), de última generación, que propicie la generación, adopción y apropiación de dicha tecnología al 
desarrollo de soluciones acordes a las necesidades reales del sector.

\section{METODOLOGÍA}

La identificación de las características del sector panelero del departamento de Boyacá parte del análisis de sus ventajas competitivas así como de las oportunidades derivadas de las políticas de impulso y participación de este sector en el producto interno bruto departamental, frente a sectores que tradicionalmente han tenido impacto en la economía del departamento, como el minero-energético, turismo y metalmecánico-metalúrgico, según lo establecido en el Plan Estratégico Departamental en Ciencia, Tecnología e Innovación de Boyacá 2022 (Ruiz, 2012).

La metodología empleada en este trabajo se fundamenta en las metodologías propuestas por Rodríguez (2011) y CIDEI (2007), en la realización de un inventario tecnológico y en la identificación de los activos tecnológicos disponibles en las empresas del sector panelero del departamento de Boyacá. Adicionalmente, se hace una búsqueda de las publicaciones o proyectos con aportes al desarrollo tecnológico de dichas empresas y que hayan sido producidos por los grupos de investigación de las universidades del departamento de Boyacá. Por último, se analiza el desarrollo e incorporación de soluciones tecnológicas en la industria panelera, cuya unidad de procesamiento principal se fundamente en dispositivos programables (microcontroladores), o en dispositivos de lógica programable (CPLD y FPGA).

\section{Identificación de sectores productivos}

Dentro del Plan Estratégico Departamental de Ciencia, Tecnología e Innovación de Boyacá PEDCTI (Ruiz, 2012), se encuentra un análisis de brechas tecnológicas que definen los sectores productivos en los que el departamento de Boyacá tiene mayor ventaja competitiva. Este análisis evalúa la actividad empresarial de cada sector para identificar tanto las capacidades existentes como las requeridas, para aumentar la competitividad en los ámbitos nacional e internacional. Los resultados de este análisis se convierten en el primer referente para identificar los sectores de interés a los cuales realizar el proceso de diagnóstico tecnológico objeto de este estudio.

Los criterios establecidos por los miembros del Concejo Departamental de Ciencia, Tecnología e Innovación (Codecti), actores económicos y equipo de trabajo del Observatorio Colombiano de Ciencia, Tecnología e Innovación (OCyT), para identificar los sectores predominantes son: "Ventajas comparativas, ventajas competitivas, sectores impulsados a través de ejercicios de política y participación en el producto interno bruto PIB Departamental" (Ruiz, 2012, p. 92).

De acuerdo con los resultados de los análisis sectoriales establecidos en el PEDCTI (Ruiz, 2012), los sectores en los que se cuenta con una ventaja comparativa son: agropecuario, explotación de minas y canteras, petrolero, artesanías y turismo histórico.

\section{Política departamental y nacional para el impulso de sectores económicos}

"Dentro de los esfuerzos departamentales por la identificación o caracterización de su estructura productiva y sus potencialidades se han realizado ejercicios que hoy constan en documentos que sirven de base para el planteamiento de políticas" (Ruiz, 2012, p. 94). La tabla 1 relaciona los documentos de política donde se establecen objetivos y retos para desarrollar ventajas competitivas y de productividad del sector productivo departamental.

En estos documentos se presenta de forma reiterada la necesidad de fomentar el desarrollo de los siguientes sectores, dadas las capacidades y fortalezas del departamento de Boyacá, de acuerdo con los enfoques y criterios de competitividad, transformación productiva y crecimiento del mercado mundial (Ruiz, 2012, p. 95). 
Tabla 1. Documentos de política sobre priorización de sectores

\begin{tabular}{|c|c|}
\hline Documento & Año \\
\hline Estudio de Competitividad para Boyacá. Araujo \& Ibarra Consultores & 1998 \\
\hline \multirow{3}{*}{$\begin{array}{l}\text { Planes de Desarrollo Departamental } \\
\text { (Londoño, 2004; Rozo, 2008; Granados, 2012) }\end{array}$} & ¡Boyacá! Deber de todos (2004-2007) \\
\hline & ¡Para seguir creciendo! (2008-2011) \\
\hline & Boyacá se atreve! (2012-2015) \\
\hline Agenda de productividad y competitividad (Gómez, 2008) & 2008 \\
\hline $\begin{array}{l}\text { Política sector agropecuario departamento de Boyacá (Secretaría de Fo- } \\
\text { mento Agropecuario, 2008) }\end{array}$ & 2008 \\
\hline $\begin{array}{l}\text { Agenda interna para la productividad y la competitividad: Documento } \\
\text { Regional Boyacá (Rentería, 2007) }\end{array}$ & 2007 \\
\hline Agenda prospectiva de Ciencia y Tecnología (Castellanos, 2004) & 2004 \\
\hline Plan regional de competitividad de Boyacá 2008-2032 (Olano, 2007) & 2007 \\
\hline $\begin{array}{l}\text { Boyacá Visión 2019: Territorio de libertad y prosperidad bicentenaria. } \\
\text { Serie: Visión de desarrollo territorial departamental }\end{array}$ & 2011 \\
\hline
\end{tabular}

Fuente: elaboración propia.

\section{Sectores con alta participación en el PIB departamental}

En la caracterización económico-productiva del departamento, para el año 2010 el PIB departamental tuvo una participación del 2,6\% en el PIB nacional, con un crecimiento promedio de $3,7 \%$ entre 2000 y 2010 , un poco menor al nacional $(4,1$ $\%)$. El PIB nacional en este año fue de $\$ 548.273$ miles de millones. A nivel de población departamental, el PIB per cápita fue de USD\$6.403, un poco menos del equivalente a nivel nacional (USD\$6.334) (Ruiz, 2012, p. 95).

El PIB del departamento da cuenta de los bienes y servicios que produce el departamento y refleja su desempeño productivo (Crepib, 2009). "En dicho año, los sectores que más aportaron al PIB departamental fueron: Actividades de servicios sociales, agropecuario, industria manufacturera, explotación de minas y canteras, transporte, almacenamiento y comunicaciones, comercio y turismo" (Ruiz, 2012, p. 95).

Una vez identificados los sectores de mayor impacto económico, el grupo de trabajo del PEDCTI (Ruiz, 2012) construyó la matriz de clasificación, donde se expone la priorización de los sectores en términos de potencial de negocios frente al entorno económico favorable. Tal clasificación se muestra en la figura 1.

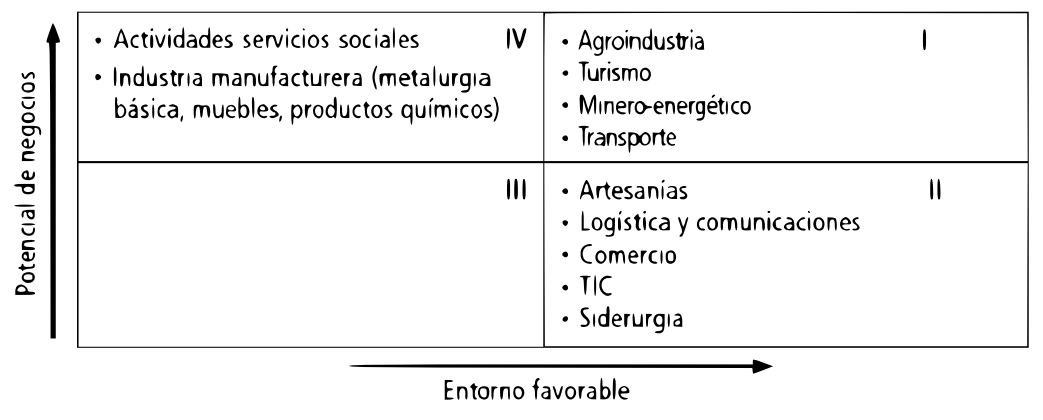

Figura 1. Matriz inicial de priorización de sectores

Fuente: Ruiz (2012). 


\section{Selección de sectores a evaluar}

La matriz construida se validó por los miembros del Codecti, los principales representantes de los sectores productivos y el equipo de trabajo del OCyT, en un ejercicio de socialización y revisión de la clasificación de los sectores ubicados en los cuatro cuadrantes de la matriz mostrada en la figura 1, y determina por consenso la distribución mostrada en la figura 2, quedando de la siguiente manera (Ruiz, 2012, p. 96).

Los sectores a analizar por parte de los actores generadores del Plan Estratégico Departamental de Ciencia, Tecnología e Innovación Boyacá 2022: la ciencia, la tecnología y la innovación al servicio del desarrollo, son los que resultaron ubicados en el cuadrante I de la matriz concertada: agroindustria, turismo, minero-energético y metalmecánica, por ser los que muestran convergencia entre su dinámica interna y el direccionamiento político. Es decir, sectores en los cuales el departamento desarrolla ventajas, además el gobierno departamental o nacional apoya a través de políticas y tienen alto potencial en el mercado (Ruiz, 2012).

\section{Criterios de selección del sector productivo panelero y realización del diagnóstico tecnológico}

El término diagnóstico es de origen griego y significa "el acto o arte de conocer", inicialmente se utilizaba en el campo de la medicina, y luego se extendió a otros ámbitos en los que también ha sido de gran utilidad, como es el caso del sector empresarial. El diagnóstico tecnológico es una herramienta de gestión que permite determinar las capacidades tecnológicas enfatizando en las fortalezas y retos por alcanzar, para lo cual se recurre al análisis de la cadena de valor examinando de forma sistemática todas las actividades que una organización desempeña y cómo interactúan entre sí (Rodríguez, 2011, p. 2-3).

La gestión de la tecnología en una organización está dividida en tres grandes partes. La primera consiste en realizar un diagnóstico interno, o tecnológico, de la empresa (inventariar y evaluar); la segunda se fundamenta en la ejecución del diagnóstico externo o diagnóstico tecnológico de los competidores, con el fin de analizar el bagaje y comportamiento de estos últimos (vigilar), y la tercera consiste en valorizar el patrimonio tecnológico de la empresa respecto a los de la competencia potencial (optimizar, enriquecer y salvaguardar) (Rodríguez, 2011, p. 3).

Para los propósitos de esta investigación, el diagnóstico tecnológico se orienta a empresas cuya actividad económica está relacionada con la producción de panela correspondiente al sector agroindustrial seleccionada en la matriz priorizada generada en el PEDCTI. El ejercicio de vigilancia aborda únicamente la fase correspondiente al diagnóstico interno para la obtención de un inventario tecnológico (máquinas, equipos y herramientas informáticas) que permita identificar los

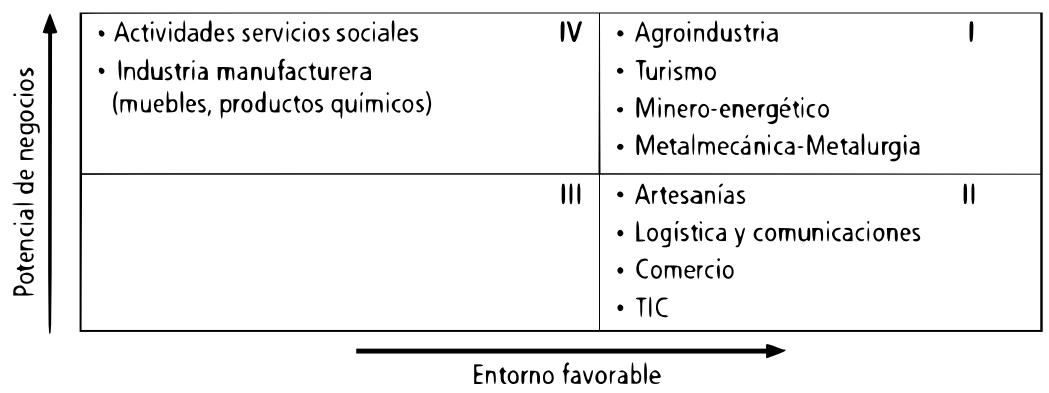

Figura 2. Matriz concertada de priorización de sectores

Fuente: Ruiz (2012). 
procesos dentro de la cadena de producción que incorporen dispositivos lógicos programables y microcontroladores.

La Gobernación de Boyacá en su página de internet dispone un directorio de las entidades cuya actividad económica corresponde al sector agroindustrial (Gobernación de Boyacá, 2014), donde se relacionan un total de 160 entidades de las cuales 18 se encuentran en la ciudad de Tunja, 13 en Duitama, 8 en Sogamoso, 6 en Paipa y en menor cuantía ubicadas en ciudades como Tibasosa, Nobsa, Puntalarga, Santana, Ventaquemada, Firavitoba, Sáchica, Samacá, Villa de Leyva, Moniquirá, Ramiriquí, Guateque, San José de Pare, Viracachá, Briceño, Toca, Tuta, entre otras. De las 17 entidades registradas en la ciudad de Tunja, 8 ya no existen (entidades liquidadas o cambio de razón social) y las direcciones no corresponden; 5 no permiten el ingreso a sus instalaciones (solo ofrecen información comercial), logrando solamente establecer contacto con 2: Secretaría de Fomento Agropecuario ubicada en las instalaciones de la Gobernación de Boyacá y el Centro Regional de Gestión para la Productividad e Innovación de Boyacá (Crepib) ubicada en las instalaciones de la Universidad Pedagógica y Tecnológica de Colombia, sede Central.

La Secretaría de Fomento Agropecuario del Departamento de Boyacá está organizada en secretarías técnicas departamentales para cada una de las cadenas productivas definidas por el Ministerio de Agricultura y Desarrollo Rural (Garavito, 2008). Mediante la Secretaría Técnica de la Cadena Productiva Agroindustrial de la Panela, se logró establecer contacto con la Coordinación Departamental de Fedepanela y el Centro de Investigación y Mejoramiento de la Panela (Cimpa-Corpoica), ubicados en el municipio de Barbosa, Santander, quienes facilitaron el acercamiento y visita a las instalaciones de fabricación de panela (trapiches) de varios productores asociados; así fue posible conocer los objetivos del proyecto de investigación, obtener información primaria del proceso del cultivo de caña de azúcar, fabricación de panela e identificación de brechas tecnológicas en su proceso de transformación.

Por otro lado,

[...] el Centro Regional de Gestión para la Productividad y la Innovación de Boyacá (Crepib) tiene como misión ser eje articulador que impulsa el mejoramiento de la productividad y competitividad a través de la innovación, el desarrollo tecnológico y la gestión del conocimiento en los sectores estratégicos de la región (Palacios, 2013, p. 192).

El Crepib EI CREPIB facilitó información y documentación respecto a la identificación de brechas tecnológicas del sector lácteo y metalmecánico, así como datos de contacto para la realización de visitas en campo, resultado de proyectos de investigación adelantados por el CREPIB; sin embargo, Crepib; sin embargo, los resultados del diagnóstico en estos sectores productivos no se describen en la presente publicación.

\section{Consideraciones para el diagnóstico tecnológico de la cadena productiva de panela}

"Reconociendo la importancia social, cultural y económica que la panela tiene en nuestro país, y con el objeto de impulsar en este sector el mejoramiento de las condiciones para la producción y la comercialización de la panela, el Instituto Nacional de Vigilancia de Medicamentos y Alimentos (Invima) y la Federación Nacional de Productores de Panela Fedepanela" (Hernández, 2009, p. 3) proporcionan los medios de divulgación de los requisitos sanitarios que deben cumplir los trapiches paneleros. Adicionalmente, la norma técnica colombiana NTC1311 (Icontec, 2009), establece los requisitos y ensayos que debe cumplir la panela destinada para el consumo humano; Osorio (2007), por su parte, presenta un instrumento orientador o manual técnico de buenas prácticas 
agrícolas (BPA) y buenas prácticas de manufactura (BPM) en la producción de caña y panela.

La metodología propuesta para la realización del diagnóstico tecnológico está basada en la metodología usada por Rodríguez (2011). De igual manera, se adopta la metodología utilizada por el Centro de Desarrollo Tecnológico de la Industria Electro Electrónica e Informática (CIDEI), donde establece una metodología de revisión de vigilancia tecnológica mediante herramientas y fuentes de búsqueda de información con buscadores de internet, metabuscadores y bases de datos (CIDEI, 2007). La figura 3 ilustra la metodología aplicada para la realización del diagnóstico tecnológico.

El inventario tecnológico es un instrumento metodológico utilizado para obtener la información cualitativa o cuantitativa sobre las tecnologías disponibles en la empresa, y posibilita la identificación de necesidades a corto, mediano o largo plazo. Un activo tecnológico se considera un equipo, herramienta o software necesario para realizar actividades productivas específicas de una empresa u organización (Rodríguez, 2011).

El análisis de incorporación tecnológica busca identificar cómo los sistemas de instrumentación electrónica, control o automatización industrial, pueden aportar al mejoramiento del proceso de producción de panela, en busca del cumplimiento de las reglamentaciones sanitarias y ambientales, así como el uso de buenas prácticas de manufactura que permitan posicionar el producto en el mercado nacional e internacional.

\section{RESULTADOS}

\section{Inventario tecnológico a trapiches y empresas visitadas}

Considerando el proceso de fabricación de panela y los parámetros establecidos como buenas prácticas agrícolas (BPA) y buenas prácticas de manufactura (BPM) (Osorio, 2007), se hace el inventario tecnológico de acuerdo con la metodología propuesta. Las empresas colaboradoras para el ejercicio de diagnóstico tecnológico son:

- Agropanela San Sebastián: Trapiche El Ingenio y Trapiche El Panelero, ubicado en la ciudad de San José de Pare.

- Doña Panela: Trapiche Doña Panela ubicadao en la ciudad de Chitaraque.

- Multingenios Makariza S.A., ubicada en el km 8, vía Barbosa-Bucaramanga. Las tablas 2 al 5 muestran los resultados del inventario tecnológico realizado en los trapiches y empresas visitadas.

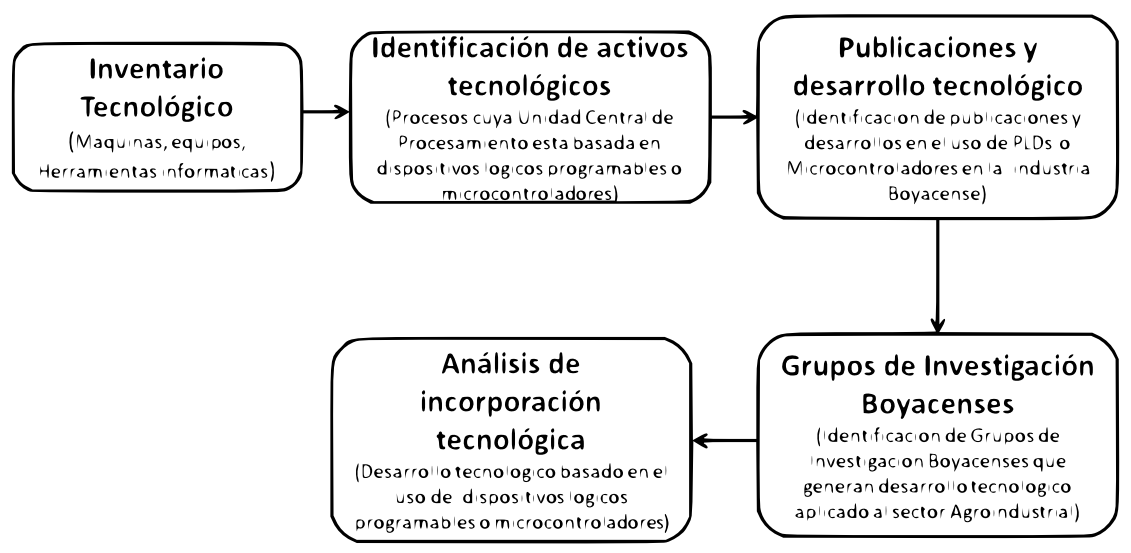

Figura 3. Metodología para diagnostico tecnológico en el sector agroindustrial de la panela

Fuente: elaboración propia. 
Tabla 2. Resultado del inventario tecnológico realizado al trapiche El Ingenio

\begin{tabular}{|c|c|c|c|}
\hline ETAPA & PROCESO & $\begin{array}{l}\text { ACTIV́O } \\
\text { TECNOLÓGICO }\end{array}$ & OBSERVACIONES \\
\hline \multirow[b]{3}{*}{ Cosecha } & Siembra & Ninguno & \multirow{3}{*}{$\begin{array}{l}\text { No hay sistema de riego. } \\
\text { No hay registro de variables climáticas y fisicoquímicas que } \\
\text { inciden sobre el cultivo. } \\
\text { No hay registro de condiciones de humedad del cultivo. } \\
\text { Cálculo del índice de madurez mediante refractómetro para } \\
\text { determinar momento de corte de la caña para beneficio. No hay } \\
\text { registro de la información. } \\
\text { El transporte se hace mediante tracción animal dada las condi- } \\
\text { ciones geográficas del lugar del cultivo de la caña de azúcar. }\end{array}$} \\
\hline & Maduración & Ninguno & \\
\hline & Corte & $\begin{array}{l}\text { Refractómetro } \\
\text { profesional, Rhb-62atc } \\
(28 \%-62 \% \text { Brix })\end{array}$ & \\
\hline \multirow{6}{*}{ Beneficio } & Apronte & Ninguno & \multirow{6}{*}{$\begin{array}{l}\text { No hay registro de cantidad de caña recolectada y dispuesta a } \\
\text { extracción. } \\
\text { No hay registro de cantidad de jugo producido en la molienda } \\
\text { respecto a cantidad de caña. } \\
\text { No hay plan de mantenimiento de molino. } \\
\text { No hay registro ni control de temperatura en los procesos de } \\
\text { evaporación y clarificación. } \\
\text { Pailas de disposición abierta. } \\
\text { El vapor de agua generada por la evaporación del guarapo dete- } \\
\text { riora los materiales de la instalación. } \\
\text { No hay registro de variables para control de calidad en el pro- } \\
\text { ceso de punteo (se hace por criterio de operario). } \\
\text { El bagazo se usa exclusivamente para alimentar hornilla. } \\
\text { Uso de hornilla tipo CIMPA [19]. }\end{array}$} \\
\hline & Extracción & $\begin{array}{l}\text { Molino con motor de } \\
\text { combustión y eléctrico. }\end{array}$ & \\
\hline & Prelimpieza & Ninguno & \\
\hline & $\begin{array}{l}\text { Clarificación y } \\
\text { encalado }\end{array}$ & Ninguno & \\
\hline & $\begin{array}{l}\text { Evaporación y } \\
\text { concentración }\end{array}$ & Ninguno & \\
\hline & $\begin{array}{l}\text { Punteo y } \\
\text { batido }\end{array}$ & Ninguno & \\
\hline $\begin{array}{l}\text { Empaque y } \\
\text { almacenamiento }\end{array}$ & Empaque & Maquina selladora & $\begin{array}{l}\text { El empaque se hace con máquina de sellado operado de mane- } \\
\text { ra manual acorde a reglamentación. }\end{array}$ \\
\hline
\end{tabular}

Fuente: elaboración propia.

Tabla 3. Resultado del inventario tecnológico realizado al trapiche El Panelero

\begin{tabular}{|c|c|c|c|}
\hline ETAPA & PROCESO & $\begin{array}{c}\text { ACTIVOO } \\
\text { TECNOLÓGICO }\end{array}$ & OBSERVACIONES \\
\hline \multirow[b]{3}{*}{ Cosecha } & Siembra & Ninguno & \multirow{3}{*}{$\begin{array}{l}\text { No hay sistema de riego. } \\
\text { No hay registro de variables climáticas y fisicoquímicas que } \\
\text { inciden sobre el cultivo. } \\
\text { No hay registro de condiciones de humedad del cultivo. } \\
\text { Cálculo del índice de madurez mediante refractómetro para } \\
\text { determinar momento de corte de la caña para beneficio. No hay } \\
\text { registro de la información. } \\
\text { El transporte se hace mediante tracción animal dada las condi- } \\
\text { ciones geográficas del lugar del cultivo. }\end{array}$} \\
\hline & Maduración & Ninguno & \\
\hline & Corte & Ninguno & \\
\hline \multirow{6}{*}{ Beneficio } & Apronte & Ninguno & \multirow{6}{*}{$\begin{array}{l}\text { No hay registro de cantidad de caña recolectada. } \\
\text { No hay registro de cantidad de jugo producido en la molienda } \\
\text { respecto a cantidad de caña. } \\
\text { No hay plan de mantenimiento de molino. } \\
\text { No hay registro ni control de temperatura en los procesos de } \\
\text { evaporación y clarificación. } \\
\text { Pailas de disposición abierta. } \\
\text { El vapor de agua generada por la evaporación del guarapo dete- } \\
\text { riora los materiales de la instalación. } \\
\text { No hay registro de variables para control de calidad en el pro- } \\
\text { ceso de punteo (se hace por criterio de operario). } \\
\text { El bagazo se usa exclusivamente para alimentar hornilla. } \\
\text { El bagazo ocupa grandes espacios para almacenaje de bagazo. } \\
\text { Tiempos prolongados para secado de bagazo para su adecuado } \\
\text { uso en la hornilla. } \\
\text { Uso de hornilla convencional. }\end{array}$} \\
\hline & Extracción & $\begin{array}{l}\text { Molino con motor de } \\
\text { combustión y eléctrico. }\end{array}$ & \\
\hline & Prelimpieza & Ninguno & \\
\hline & $\begin{array}{l}\text { Clarificación y } \\
\text { encalado }\end{array}$ & Ninguno & \\
\hline & $\begin{array}{l}\text { Evaporación y } \\
\text { concentración }\end{array}$ & Ninguno & \\
\hline & $\begin{array}{l}\text { Punteo y } \\
\text { batido }\end{array}$ & Ninguno & \\
\hline $\begin{array}{l}\text { Empaque y al- } \\
\text { macenamiento }\end{array}$ & Empaque & Ninguna & No dispone de espacio para almacenamiento y empaque. \\
\hline
\end{tabular}

Fuente: elaboración propia. 
Tabla 4. Resultado del inventario tecnológico realizado al trapiche Doña Panela

\begin{tabular}{|c|c|c|c|}
\hline ETAPA & PROCESO & $\begin{array}{l}\text { ACTIV́O } \\
\text { TECNOLÓGICO }\end{array}$ & OBSERVACIONES \\
\hline \multirow[b]{3}{*}{ Cosecha } & Siembra & Ninguno & \multirow{3}{*}{$\begin{array}{l}\text { No hay sistema de riego. } \\
\text { No hay registro de variables climáticas y fisicoquímicas del } \\
\text { suelo que inciden sobre el cultivo. } \\
\text { No hay registro de condiciones de humedad del cultivo. } \\
\text { Cálculo del índice de madurez mediante refractómetro para } \\
\text { determinar momento de corte de la caña para beneficio. No hay } \\
\text { registro de la información. } \\
\text { El transporte se hace mediante tracción animal dada las condi- } \\
\text { ciones geográficas del lugar del cultivo de la caña de azúcar. }\end{array}$} \\
\hline & Maduración & Ninguno & \\
\hline & Corte & $\begin{array}{l}\text { Refractómetro portátil } \\
\text { de } 45-82^{\circ} \text { Brix marca } \\
\text { Scientific }\end{array}$ & \\
\hline \multirow{6}{*}{ Beneficio } & Apronte & Ninguno & \multirow{6}{*}{$\begin{array}{l}\text { Se hace medición de cantidad de caña recolectada y dispuesta } \\
\text { a extracción pero no hay registro. } \\
\text { No hay registro de cantidad de jugo producido en la molienda } \\
\text { respecto a cantidad de caña. } \\
\text { No hay plan de mantenimiento de molino. } \\
\text { No hay registro ni control de temperatura en los procesos de } \\
\text { evaporación y clarificación. } \\
\text { Pailas de disposición abierta. } \\
\text { El vapor de agua generada por la evaporación del guarapo dete- } \\
\text { riora los materiales de la instalación. } \\
\text { No hay registro de variables para control de calidad en el proce- } \\
\text { so de punteo (se hace por criterio de operario). } \\
\text { El bagazo se usa exclusivamente para alimentar hornilla. } \\
\text { Uso de hornilla tipo CIMPA. }\end{array}$} \\
\hline & Extracción & $\begin{array}{l}\text { Molino con motor de } \\
\text { combustión y eléctrico. }\end{array}$ & \\
\hline & Prelimpieza & Ninguno & \\
\hline & $\begin{array}{l}\text { Clarificación y } \\
\text { encalado }\end{array}$ & Ninguno & \\
\hline & $\begin{array}{l}\text { Evaporación y } \\
\text { concentración }\end{array}$ & Ninguno & \\
\hline & $\begin{array}{l}\text { Punteo y } \\
\text { batido }\end{array}$ & Ninguno & \\
\hline $\begin{array}{l}\text { Empaque y } \\
\text { almacenamiento }\end{array}$ & Empaque & Máquina selladora & $\begin{array}{l}\text { El empaque se hace con máquina de sellado operado de mane- } \\
\text { ra manual acorde a reglamentación. }\end{array}$ \\
\hline
\end{tabular}

Fuente: elaboración propia.

Tabla 5. Resultado del inventario tecnológico realizado Multingenios Makariza S.A.

\begin{tabular}{|c|c|c|c|}
\hline ETAPA & PROCESO & $\begin{array}{c}\text { ACTIV́O } \\
\text { TECNOLÓGICO }\end{array}$ & OBSERVACIONES \\
\hline \multirow{3}{*}{ Cosecha } & Siembra & Indeterminado & \multirow{3}{*}{$\begin{array}{l}\text { Disponen de diferentes proveedores de caña de azúcar. } \\
\text { Fomento a la calidad de materia prima, aumentando el pago } \\
\text { de } \$ / \text { ton por cada grado Brix (nivel de sacarosa) por encima del } \\
\text { promedio de la zona que es de } 19 \text { grados. } \\
\text { Cuenta actualmente con } 150 \text { proveedores con radio de cercanía } \\
\text { de } 9 \mathrm{~km} \text { para no aumentar costos de transporte. }\end{array}$} \\
\hline & Maduración & Indeterminado & \\
\hline & Corte & Indeterminado & \\
\hline \multirow[t]{4}{*}{ Beneficio } & Apronte & $\begin{array}{l}\text { Báscula para pesaje de } \\
\text { materia prima }\end{array}$ & $\begin{array}{l}\text { La planta cuenta con monitoreo permanente de ingenieros de } \\
\text { producción y revisores de calidad. } \\
\text { Vapor de caldera destinado para alimentar difusor y generación } \\
\text { de energía proyectado para generador eléctrico de } 50 \mathrm{KVA} \text {. } \\
\text { Alguna información de registro no revelada por políticas de } \\
\text { protección de información. }\end{array}$ \\
\hline & Trituración & Picadora DNIndustrial & \\
\hline & Difusión & $\begin{array}{l}\text { Difusor de caña de azú- } \\
\text { car DNIndustrial }\end{array}$ & \\
\hline & Caldera & $\begin{array}{l}\text { Caldera de vapor DNIn- } \\
\text { dustrial }\end{array}$ & \\
\hline $\begin{array}{l}\text { Empaque y al- } \\
\text { macenamiento }\end{array}$ & Empaque & Indeterminado & $\begin{array}{l}\text { Empaque en bolsas de polietileno en presentaciones que van des- } \\
\text { de } 6 \text { g, } 500 \text { g, } 1000 \text { g y } 2500 \text { g de panela pulverizada natural. } \\
\text { Bodegas en Bogotá y próximamente Medellín y Barranquilla. }\end{array}$ \\
\hline
\end{tabular}

Fuente: elaboración propia. 
La Federación Nacional de productores de Panela (Fedepanela) informa que los departamentos con mayor producción de panela son Santander, Antioquia, Cundinamarca y Boyacá, y solamente en Boyacá el sector panelero presenta un alto grado de informalidad: 13.769 establecimientos productores son empresas sin constitución legal (Hernández, 2009). La Dirección de Fedepanela comenta que lo ideal es implementar procesos de producción con valor agregado como el mostrado por la empresa Multingenios Makariza S.A., pero el alto costo de la tecnología necesaria impide su adopción y transferencia, que el esfuerzo de las directivas de Fedepanela y a legislación nacional es lograr que los trapiches cumplan estándares mínimos como el caso presentado por el Trapiche El Ingenio y Doña Panela, pero la realidad del sector productivo de los trapiches del sector de la hoya del río Suárez está reflejado en lo diagnosticado en el Trapiche El Panelero. Por tal razón, la directora de Fedepanela recomienda En entrevista realizada el día 6/Marzo/2014 a la coordinadora departamental de Fedepanela, Magda Liliana Murcia Pardo, da la recomendación de "no desgastarse en tantas visitas, pues encontraremos la misma situación que la vista en el trapiche El Panelero, y seguramente procesos más precarios".

\section{Publicaciones en el sector agroindustrial de Boyacá}

Con la metodología propuesta por el CIDEI para la identificación de publicaciones en fuentes de información, como bases de datos y metabuscadores (CIDEI, 2007), las bases de datos utilizadas para la búsqueda de publicaciones relacionadas con la aplicación de tecnología basada en el uso de dispositivos lógicos programables y microcontroladores en la industria boyacense son: IEEE Xplore y Science Direct, dado que la Universidad Pedagógica y Tecnológica de Colombia cuenta con licencia para el uso de estas bases de datos. Adicionalmente, se recurre a Google Academic de dominio público y gratuito. Para el registro de la información se consulta una tabla que identifica la base de datos, la ecuación de búsqueda, número de registros encontrados, número de artículos con pertinencia y fecha de búsqueda. Los resultados que arroja la búsqueda en la base de datos IEEE Xplore, correspondientes a la aplicación de microcontroladores, CPLD y FPGA en sistemas de control, monitoreo y procesos de tecnificación en la agroindustria, se muestran en la tabla 6.

De un total de 203 registros, solamente 9 publicaciones fueron pertinentes acorde al objetivo de la búsqueda. A continuación, se hace una breve descripción de las publicaciones identificadas.

En aplicaciones de microcontroladores en la industria agrícola, Aravinth (2012) describe el uso de máquinas inteligentes agrícolas para los procesos de prueba y enriquecimiento de suelos, siembra de semillas, monitoreo de condiciones climáticas y comprobación de estructuras de ADN en las plantas, para determinar la aplicación de nutrientes en caso de carencia. También, Jie (2011) plantea el desarrollo de un sistema de control Fuzzy-PID basado en microcontrolador para controlar los motores como elementos actuadores y procesamiento de las señales de los sensores que determinan la posición de siembra de semillas de trigo. De igual manera, Chaitanya (2007) expone el diseño de un sistema de adquisición de datos datalogger para $\mathrm{pH}$ usando microcontrolador, explicando su uso en áreas como bioquímica, agronomía, ciencias de los alimentos y control ambiental .

Para el caso del uso de FPGA, se registró un solo documento correspondiente al trabajo realizado por Jayendra (2009), donde presenta el diseño de un control avanzado basado en FPGA para el proceso de siembra.

Por último, orientando la búsqueda al uso de microcontroladores en sistemas de control, monitoreo y tecnificación, se encontraron trabajos como el de Gutiérrez (2014), quien elabora un sistema de riego automatizado para uso de agua en cultivos agrícolas. Sylvain describe el desarrollo de algoritmos de criptografía usando microcontroladores para disminución de consumo de energía 
(Pelissier, 2011). Sánchez (2011) desarrolla un sistema de redes inalámbricas de sensores subacuáticos UWSN para uso en áreas de acuicultura, exploración marina y control biológico para aguas contaminadas, fondo marino, corrientes oceánicas y actividad sísmica. Chunyan (2010) describe un sistema de flujo ultrasónico inalámbrico mediante microcontroladores para aprovechamiento de la energía en sistemas automotrices. Porotro lado, Zhi (2010) desarrolla una red de sensores inalámbrica para monitoreo remoto del medio ambiente a través de varios protocolos de comunicación.

Los resultados encontrados en la base de datos Science Direct (Elsevier) correspondientes a la aplicación de microcontroladores, CPLD y FPGA en sistemas de control, monitoreo y procesos de tecnificación en la agroindustria, se muestran en la tabla 7.

Se toma al microcontrolador como dispositivo programable clave en la ecuación de búsqueda, considerando la tendencia obtenida en los registros de Science Direct. De un total de 144 registros, solamente 6 fueron pertinentes. A continuación, se hace una breve descripción de las publicaciones identificadas.

Para aplicaciones de los microcontroladores en el sector agroindustrial, Escolar (2011) describe una metodología novedosa para el monitoreo de laproducción agrícola, basada en red de sensores inalámbrica. Zhua (2011) plantea unas aplicaciones de redes de sensores inalámbrica para el monitoreo de ambientes agrícolas, principalmente supervisión de temperatura y humedad. De igual manera, López (2009) muestra el desarrollo de un sistema preciso de redes de sensores inalámbrica para cultivos hortícolas en el norte de España, obteniendo mejoras en la administración del recurso hídrico y mejoramiento del proceso de fertirrigacion. Zafar (2011) exponeuna experiencia durante la introducción del desarrollo experimental de redes de sensores en una empresa de producción hortícola ecológica en la región de Murcia España. Zhanga (2008) sugier un sistema de predicción de acidez, solidos solubles y firmeza usando técnica de nariz electrónica. Aquí describen algoritmos de métodos de calibración multivariable, regresión lineal múltiple $M L R$, regresión de componente lineal PCR y regresión parcial de media cuadrática PLS. Por último, para aplicaciones específicas en las mediciones de variables tanto físicas como químicas en la producción de panela, con microcontroladores, se encontró una publicación realizada por García (2011) quien hace un estudio comparativo de algoritmos de reconocimiento de patrones implementados en microcontrolador para usar en una lengua electrónica para monitoreo de agua potable, cuyo producto objetivo no es propiamente la panela, pero el método aplica para las condiciones requeridas en los procesos BPA y BPM de la panela.

Los resultados encontrados en la base de datos Google Academic correspondientes a la aplicación de microcontroladores, CPLD y FPGA en sistemas de control, monitoreo y procesos de tecnificación en la producción de panela, se muestran en la tabla 8 .

Tabla 7. Uso de microcontroladores en aplicaciones de agroindustria usando Science Direct

\begin{tabular}{llccc}
\hline $\begin{array}{c}\text { Herramienta de } \\
\text { búsqueda }\end{array}$ & \multicolumn{1}{c}{ Descriptores } & $\begin{array}{c}\text { No. de } \\
\text { registros }\end{array}$ & $\begin{array}{c}\text { Pertinencia } \\
\text { del resultado }\end{array}$ & $\begin{array}{c}\text { Fecha de } \\
\text { búsqueda }\end{array}$ \\
\hline \multirow{3}{*}{ ScienceDirect } & agricultural industry application microcontrollers & 21 & 4 & $3 / 29 / 2014$ \\
\cline { 2 - 5 } & $\begin{array}{l}\text { chemical measurement of physical conditions for panela } \\
\text { chemical measurement of physical conditions using } \\
\text { microcontrollers }\end{array}$ & 44 & 1 & $3 / 29 / 2014$ \\
\hline
\end{tabular}

Fuente: elaboración propia. 
Tabla 8. Ahorro energético y tecnificación de la producción de panela usando Google Academic

\begin{tabular}{clccc}
\hline $\begin{array}{c}\text { Herramienta de } \\
\text { búsqueda }\end{array}$ & \multicolumn{1}{c}{ Descriptores } & $\begin{array}{c}\text { No. de } \\
\text { registros }\end{array}$ & $\begin{array}{c}\text { Pertinencia } \\
\text { del resultado }\end{array}$ & $\begin{array}{c}\text { Fecha de } \\
\text { búsqueda }\end{array}$ \\
\hline \multirow{2}{*}{ Google academic } & $\begin{array}{l}\text { ahorro energético en producción de panela, tecnifi- } \\
\text { cación en producción de panela }\end{array}$ & 714 & 6 & $3 / 29 / 2014$ \\
\cline { 2 - 5 } & mejoramiento en producción de panela & 683 & 3 & $3 / 30 / 2014$ \\
\hline
\end{tabular}

Fuente: elaboración propia.

De 1397 registros, se clasificaron solamente 9 publicaciones orientadas al ahorro energético en producción de panela y su mejoramiento. A continuación, se hace una breve descripción de las publicaciones identificadas:

Flórez (2013) expone la agenda prospectiva de la cadena productiva de la panela y su agroindustria. Mendieta (2011) evalúa la eficiencia de pailas en hornos para la elaboración de panela. Ordóñez (2007) realiza el modelamiento de la producción de panela usando MatLab y orientado a control de procesos industriales principalmente a concentración de azúcar y el nivel en los efectos del evaporador. Por su parte, López (2009) diseña e implementa un prototipo de sistema de control basado en microcontrolador para monitoreo de temperatura y apertura de válvulas para flujo de jugos entre pailas. Rosero (2011) diagnostica la producción, transformación y comercialización de panela en cabildos indígenas nasa del norte del Cauca. Gallego (2013) investiga los trapiches del municipio de Santuario, Risaralda. León (2013) desarrolla una máquina de empacado y control de peso para panela granulada. Los documentos anteriores corresponden a proyectos de grado disponibles en la red Google Academic y no corresponden a documentos publicados en revistas indexadas o de divulgación científica reconocida.

Por otro lado, Mujica (2008) muestra un análisis del efecto de la variedad, lavado de la caña y temperatura de punteo sobre la calidad de la panela granulada. Loyo (2002) expone algunos métodos y estrategias para el perfeccionamiento de la agroindustria panelera, identificando problemas de rendimiento de cultivo, perdida de extracción de jugos, ineficiencia térmica en las hornillas y deficiencia en la calidad del producto final. Guaman (2009) realiza el diseño, simulación y emulación de una planta productora de panela, incorporando elementos de automatización de procesos para mejoramiento de condiciones en las actividades de los operarios y mejoramiento del recurso energético. De las investigaciones mencionadas, se resalta que ninguna fue realizada en Colombia, pues corresponden a investigaciones realizadas en países como Ecuador y Costa Rica.

\section{Grupos de investigación con publicaciones de aplicación de dispositivos programables para la agroindustria boyacense}

Considerando que la misión de las universidades del departamento de Boyacá obedece principalmente a cubrir las necesidades del entorno, se realizó la investigación de los grupos de investigación reconocidos y categorizados por Colciencias, que desarrollan aplicaciones de control, instrumentación, monitoreo y automatización de procesos agroindustriales, siendo estas áreas de aplicación las identificadas en la revisión documental encontrada en las bases de datos utilizadas en la metodología de diagnóstico tecnológico. La fuente primaria es la información registrada en GrupLac de los grupos de investigación. Las cinco principales universidades del departamento de Boyacá son: Santo Tomas, Pedagógica y Tecnológica de 
Colombia (UPTC), de Boyacá, Fundación Universitaria Juan de Castellanos, y Antonio Nariño. La búsqueda de los grupos de investigación se limita al área de conocimiento de ingeniería eléctrica, electrónica e informática, de lo cual se encontró lo relacionado en la tabla 9.

Se identificó un total de 8 publicaciones correspondientes a la electrónica, control, sistemas de información, robótica, sistemas inteligentes e instrumentación aplicadas al sector agroindustrial.

Cavieles (2012) propone el desarrollo de un modelo de autómata finito, como alternativa de solución para la automatización en la elaboración del yogur en pymes. Jiménez (2006) expone el desarrollo de sistemas de agricultura de precisión para la planificación mediante sistemas de información geográfica. De igual manera, Torres (2009) caracteriza la firma espectral a partir de sensores remotos para el manejo de sanidad vegetal en el cultivo de palma de aceite. Gómez (2005) desarroIla una estación agrometereológica y agroclimática automática para la supervisión de variables que apoyan la toma de decisión en cultivos en la zona de la región del Sugamuxi. Por último, Jiménez (2010) propone un sistema de adquisición, almacenamiento y análisis de información fenológica para el manejo de plagas y enfermedades de un duraznero mediante tecnologías de agricultura de precisión. Jiménez (2012) muestra el desarrollo de un sistema de telemetría de temperatura en cultivos agrícolas usando software libre, y expone además un sistema para el monitoreo de variables en campo en tiempo real .

Tabla 9. Publicaciones realizadas por las principales universidades del departamento de Boyacá

\begin{tabular}{|c|c|c|c|c|c|}
\hline Institución & $\begin{array}{c}\text { Grupos de } \\
\text { investigación }\end{array}$ & $\begin{array}{l}\text { Grupos de investigación } \\
\text { por area de conocimiento }\end{array}$ & Categoría & $\begin{array}{c}\text { Líneas de } \\
\text { investigación }\end{array}$ & $\begin{array}{c}\text { Publicaciones } \\
\text { pertinentes }\end{array}$ \\
\hline $\begin{array}{l}\text { Universidad } \\
\text { Santo Tomas } \\
\text { Tunja }\end{array}$ & 7 & $\begin{array}{l}\text { Grupo de Investigación y } \\
\text { Desarrollo de Ingeniería en } \\
\text { nuevas Tecnologías (GIDINT) }\end{array}$ & B & Instrumentación y control & 2 \\
\hline \multirow{2}{*}{$\begin{array}{l}\text { Universidad } \\
\text { Pedagógica y } \\
\text { Tecnológica } \\
\text { de Colombia } \\
\text { (UPTC) }\end{array}$} & \multirow[t]{2}{*}{102} & $\begin{array}{l}\text { Grupo de Investigación en } \\
\text { Procesamiento de Señales } \\
\text { DSP-UPTC }\end{array}$ & Reconocido & $\begin{array}{l}\text { Electrónica de potencia. } \\
\text { Instrumentación y Automati- } \\
\text { zación industrial. } \\
\text { Procesamiento de señales. } \\
\text { Tecnologías agropecuarias. }\end{array}$ & 5 \\
\hline & & $\begin{array}{l}\text { Grupo de Investigación en } \\
\text { Robótica y Automatización } \\
\text { Industrial (GIRA) }\end{array}$ & C & $\begin{array}{l}\text { Automatización industrial. } \\
\text { Inteligencia artificial. } \\
\text { Sistemas embebidos. } \\
\text { Robótica. }\end{array}$ & 0 \\
\hline $\begin{array}{l}\text { Universidad de } \\
\text { Boyacá }\end{array}$ & 18 & $\begin{array}{l}\text { Grupo de Investigación en } \\
\text { Instrumentación, Modela- } \\
\text { miento, Automatización y } \\
\text { Control }\end{array}$ & $\mathrm{D}$ & $\begin{array}{l}\text { Robótica, automatización y } \\
\text { control. } \\
\text { Instrumentación electrónica. }\end{array}$ & 1 \\
\hline $\begin{array}{l}\text { Fundación Uni- } \\
\text { versitaria Juan } \\
\text { de Castellanos }\end{array}$ & 9 & $\begin{array}{l}\text { Grupo de Investigación en In- } \\
\text { formática Avanzada-MUISCA }\end{array}$ & C & $\begin{array}{l}\text { Ingeniería web. } \\
\text { Procesos de software y siste- } \\
\text { mas de control. } \\
\text { Seguridad informática y de la } \\
\text { información. } \\
\text { Sistemas inteligentes. }\end{array}$ & 0 \\
\hline $\begin{array}{l}\text { Universidad } \\
\text { Antonio Nariño }\end{array}$ & 29 & $\begin{array}{l}3 \\
\text { (Ninguna en Boyacá) }\end{array}$ & Ninguna & ( & 0 \\
\hline
\end{tabular}

Fuente: elaboración propia. 


\section{Análisis de incorporación tecnológica al sector productivo agroindustrial de Boyacá}

De acuerdo con los resultados obtenidos en el ejercicio de diagnóstico tecnológico realizado en las empresas productoras de panela: El Ingenio, El Panelero y Doña Panela, empresas productivas de panela del sector de la hoya de río Suárez, existen amplias posibilidades para desarrollo tecnológico basado en dispositivos lógicos programables y microcontroladores para el fortalecimiento en los eslabones de producción primaria y transformación del sector productivo panelero de Boyacá. Tales desarrollos deben estar orientados a procesos como:

- Sistemas de control de riego para conservación de recursos hídricos.

- Sistemas autómatas de fertirrigación o administración de fertilizantes según requerimientos de cultivo.

- Monitoreo de variables climatológicas.

- Monitoreo de variables para análisis fitosanitario.

- Monitoreo de variables de proceso para trazabilidad de producto.

- Sistemas de control e instrumentación para el desarrollo de energías alternativas aprovechando residuos de cosecha y poscosecha.

- Sistemas de control y automatización para mejoramiento de condiciones laborales.
Las anteriores consideraciones se complementan según lo diagnosticado también en el Plan Estratégico Departamental de Ciencia Tecnología e Innovación de Boyacá, en el que se define la estructura de la agroindustria del departamento de Boyacá "como una economía primaria con intentos individuales y aislados de procesos de agregación de valor a los productos primarios, escaza utilización de tecnología importante en sus procesos industriales y niveles bajos de inserción en el mercado global" (Ruiz, 2012). En términos generales, se podrían establecer las razones mostradas en la tabla 10, como el argumento para desarroIlar tecnología al alcance del pequeño y mediano productor de panela, considerando los dispositivos lógicos programables y los microcontroladores como una alternativa para tal fin.

Por último, dadas las tendencias mundiales de innovación en el sector agroindustrial, teniendo en cuenta inicialmente el eslabón de producción primaria de la panela, se establece la necesidad de incorporar sistemas de mecanización en la siembra y cosecha de la caña panelera, utilización de tecnologías para el desarrollo de la agricultura de precisión, mejoramiento fitosanitario, manejo de los recursos naturales y prevención de plagas y malezas del cultivo. Para el eslabón de transformación o beneficio de la caña panelera la optimización del proceso de conversión de la panela, procesos de

Tabla 10. Problemas y desafíos del sector agroindustrial de Boyacá

\begin{tabular}{|c|c|}
\hline PRODUCCIÓN PRIMARIA & TRANSFORMACIÓN \\
\hline $\begin{array}{l}\text { Bajos niveles de certificación en temas de calidad, ambienta- } \\
\text { les y de inocuidad }\end{array}$ & $\begin{array}{l}\text { Se ha incorporado escasa tecnología e innovación en la } \\
\text { industria. }\end{array}$ \\
\hline $\begin{array}{l}\text { Bajos niveles de adopción/adaptación tecnológica y biotecno- } \\
\text { lógica en todo el sector. }\end{array}$ & $\begin{array}{l}\text { Niveles básicos o inexistentes de utilización de los desechos } \\
\text { y subproductos para la generación de nuevos productos y } \\
\text { disminución de costos. }\end{array}$ \\
\hline Bajos niveles de disponibilidad de información estadística. & $\begin{array}{l}\text { Bajos niveles de innovación en sistemas de ahorro de energía } \\
\text { y disminución de costos en procesos de transformación. }\end{array}$ \\
\hline $\begin{array}{l}\text { Pequeños y medianos productores con baja capacidad organi- } \\
\text { zacional, y resistentes a cambios tecnológicos y utilización de } \\
\text { nuevas técnicas/tecnologías. }\end{array}$ & $\begin{array}{l}\text { Alta desarticulación entre agentes sectoriales, académicos y } \\
\text { estatales. }\end{array}$ \\
\hline
\end{tabular}

Fuente: Ruiz (2012). 
automatización para mejoramiento de las condiciones laborales en la producción de panela, manejo y aprovechamiento de la biomasa residual, identificación y mejoramiento del proceso sanitario e incorporación de energías alternativas a través del aprovechamiento de la biomasa residual (tabla 11).

Tabla 11. Tendencias mundiales de innovación para el sector agroindustrial

\section{PRODUCCIÓN PRIMARIA}

Sensores remotos e imágenes satelitales para detección de deficiencias fisiológicas y para control de malezas y fumigación.

Robots y mecanización para cosecha de fruto y fertilización.

Requerimientos y suelos aptos para el cultivo. Aumento en rendimiento por área cultivada.

Manejo eficiente (tecnificado) del recurso hídrico.

\section{TRANSFORMACIÓN}

Aplicación de tecnologías y procedimientos más limpios.

Investigación básica y aplicada en transformación de productos e inocuidad.

Uso eficiente de la energía en plantas de beneficio.

Promoción de energías alternativas a partir de biomasa y reutilización de residuos de procesos de cosecha y poscosecha.

Fuente: Ruiz (2012).

\section{CONCLUSIONES}

Multingenios Makariza S.A. incorpora tecnología de punta para la producción de panela pulverizada, tecnología basada en difusores que permite un mayor aprovechamiento de la caña panelera y obtención de subproductos derivados. Los trapiches El Ingenio y Doña Panela incorporan, en su cadena de producción, hornillas tipo CIMPA para mejoramiento térmico y aprovechamiento residual. Sin embargo, no cuentan con sistemas de información en los eslabones de producción y beneficio, solamente usan algunos elementos como refractómetros para medición de grados Brix. El caso más crítico se evidenció en el trapiche El Panelero, pues no cuenta con ningún sistema de información del producto, la hornilla es tradicional, el bagazo como combustible requiere periodos prolongados de secado y el proceso es manual, sin ningún instrumento de apoyo para garantizar la calidad de producto. El aprovechamiento de residuo como combustible y los consumos excesivos de energía eléctrica en la producción de panela es un común denominador en los trapiches. Por tanto, no se identificó ningún sistema basado en dispositivos lógicos programables y microcontroladores en estas tres empresas.
A través de IEEE Xplore, Science Direct y Google Academic, se encontraron aplicaciones con microcontroladores, CPLD y FPGA en sistemas automáticos de siembra, sistemas de riego, registro de variables climáticas en cultivo, agricultura de precisión, redes de sensores para medición de variables en cultivo y técnicas avanzadas, como lengua electrónica y nariz electrónica, para determinar características físicas y químicas de producto. Ninguno de los desarrollos se orienta a la producción de panela, sin embargo, son tecnologías que pueden ser adoptadas y apropiadas a este sector agroindustrial.

Por último, la Universidad Pedagógica y Tecnológica de Colombia y la Universidad Santo Tomas son líderes en el desarrollo de aplicaciones tecnológicas orientadas a la agroindustria boyacense, ninguna está enfocada en la cadena productiva, sin embargo, son escalables a esta.

\section{FINANCIAMIENTO}

Los resultados descritos se derivan del proyecto de investigación "Diagnóstico tecnológico del uso de dispositivos lógicos programables y microcontroladores en la industria boyacense", financiado por la Dirección de Investigaciones de la Universidad 
Pedagógica y Tecnológica de Colombia, dentro del marco de la convocatoria DIN017 de 2014, para la vinculación de docentes a grupos de investigación.

\section{REFERENCIAS BIBLIOGRÁFICAS}

Aravinth, G. (2012). Wedding of Robots with Agriculture. Third International Conference on Computing Communication \& Networking Technologies (ICCCNT), 4.

Castellanos, C. (2004). Agenda prospectiva de Ciencia y Tecnología de Boyacá. Tunja: Colciencias, SENA.

Cavieles, N. (2012). Modelo de autómata finito orientado a mejorar la productividad en el procesamiento de yogur Colombia. Revista Educación en Ingeniería, 7(13), 63-73.

Chaitanya, S. (2007). Designing a pH Data Acquisition and Logging Device using an Inexpensive Microcontroller. Proceedings Southeast Conference IEEE, 217-220.

Chunyan, M. (2010). Research on a Self-powered Wireless Ultrasonic Flow Sensor System. Conference on Progress in Informatics and Computing (PIC), 2010 IEEE International, 522-526.

Centro de Desarrollo Tecnológico de la Industria Electro Electrónica e Informática (CIDEI) (2007). Metodología de Vigilancia Tecnológica: Informe tipo vigilancia científico técnica. Bogotá: Centro de Investigación y Desarrollo Tecnológico de la Industria Electro Electrónica e Informática.

Centro Regional de Gestión para la Productividad y la Innovación de Boyacá (CREPIB) (2009). Boyacá, agroindustria productiva y competitiva. Boletín, 1. Tunja: Centro Regional de Gestión para la Productividad y la Innovación de Boyacá.

Escolar, S. (2011). A novel methodology for the monitoring of the agricultural production process based on wireless sensor networks. (Elsevier, Ed.) Computers and Electronics in Agriculture, 252-265.

Flórez, D.H. (2013). Agenda prospectiva de investigación de la cadena productiva de la panela y su agroindustria. Revista Tecnura, 17(36), 72-86.

Gallego, J. (2013). Investigación de los trapiches del municipio de santuario Risaralda, análisis de productividad y oportunidades de mejora para la competitividad del sector. Universidad Tecnológica de Pereira, Facultad de Ingeniería Industrial, Pereira.

Garavito, J. (2008). Cartilla cadenas productivas. Bogotá D.C.: Ministerio de Agricultura y Desarrollo Rural.

García, E. (2011). A comparison study of pattern recognition algorithms implemented on a microcontroller for use in an electronic tongue for monitoring drinking waters. (Elsevier, Ed.) Journal Sensors and Actuators a Physical, vol. 172, issue 2, 570-582.

Gobernación de Boyacá (2014).Directorio agroindustrial. Recuperado el 31 de julio de 2014, de: http:// www.boyaca.gov.co/images/Directorios/DIRECTORIO\%20AGROINDUSTRIAL.pdf

Gómez, A. (2005). Estaciones agrometeorológicas y agroclimáticas automáticas. Revista Ingeniería, Investigación y Desarrollo, vol. 5, No. 1, 37-42.

Gómez, E.H. (2008). Agenda interna para la productividad y competitividad. Tunja: Secretaria Técnica de la Región Central de Colombia.

Granados, J.C. (2012). Plan de desarrollo departamental de Boyacá 2012-2015. Tunja: Gobernación de Boyacá.

Guaman, F. (2009). Diseño, Simulación y Emulación de una Planta Productora de Panela. Facultad de Ingeniería en Electricidad y Computación (FIEC). Ecuador, disponible en: http://www.panelamonitor. org/media/docrepo/document/files/diseno-simulacion-y-emulacion-de-una-planta-de-produccion-de-panela.pdf.

Gutiérrez, J. (2014). Automated Irrigation System Using a Wireless Sensor Network and GPRS Module. IEEE Transactions on Instrumentation and Measurement, 63(1), 166-176.

Hernández, L. (2009). ABC Panela. Bogotá: Ministerio de la Protección Social, Invima, Ministerio de Agricultura y Desarrollo Rural y Fedepanela.

Instituto Colombiano de Normas Técnicas (Icontec) (2009). Norma Técnica Colombiana NTC 1311: Productos agrícolas PANELA. Bogota: Icontec.

Jayendra, K. (2009). FPGA based advanced sowing and planting equipment controller design. International Conference on Emerging Trends in Electronic 
and Photonic Devices \& Systems ELECTRO '09, 120-123.

Jie, L. (2011). Study on the Application of Fuzzy-PID Control to Precision Seeding Technology of Wheat. Second International Conference on Mechanic Automation and Control Engineering (MACE), 1690-1693.

Jiménez, A. (2006). Sistemas de agricultura de precisión planificación mediante sistemas de información geográfica. Revista Ingeniería, Investigación y Desarrollo, vol. 5, No. 2, 44-47.

Jiménez, A. (2010). Sistema de adquisición, almacenamiento y análisis de información fenológica para el manejo de plagas y enfermedades de un duraznero mediante tecnologías de agricultura de precisión. Revista Tecnura, 1(1), 41-51.

Jiménez, F. (2012). Field variables monitoring in real time (GPS, soil moisture, temperature) with precision farming applications. EATIS '12: Proceedings of the 6th Euro American Conference on Telematics and Information Systems, 367-370.

Jiménez, F. (2012). Temperature telemetry system for agricultural crops using free software. EATIS '12: Proceedings of the 6th Euro American Conference on Telematics and Information Systems, 89-94.

León, G. (2013). Máquina de empacado y control de peso para panela granulada. Universidad Técnica del Norte, Ecuador, disponible en: http://www. panelamonitor.org/media/docrepo/document/files/ maquina-de-empacado-y-control-de-peso-para-panela-granulada.pdf.

Londoño, J.E. (2004). Plan de desarrollo departamental de Boyacá 2004-2007. Tunja: Gobernación de Boyacá.

López, J. (2009). Instrumentación electrónica, aplicada en el proceso de producción de panela, enfocada a microempresarios. Bogotá: Corporación Universitaria Minuto de Dios. Disponible en: http://repository.uniminuto.edu:8080/xmlui/handle/10656/2695.

López, J. (2009). Wireless Sensor Networks for precision horticulture in Southern Spain. (Elsevier, Ed.) Computers and Electronics in Agriculture, 45-52.

Loyo, R. (2002). Métodos y estrategias para el perfeccionamiento de la agroindustria panelera. Disponible en: http://www.panelamonitor.org/media/docrepo/ document/files/metodos-y-estrategias-para-el-perfeccionamiento-de-la-agroindustria-panelera.pdf

Mendieta, O. (2011). Ahorro de combustible y energía en hornos usados para la elaboración de panela modificando el diseño de pailas abiertas. Revista Fuentes, 9, 53-61.

Mujica, M. (2008). Efecto de la variedad, lavado de la caña y temperatura de punteo sobre la calidad de la panela granulada. Revista Interciencia, vol 33, No. 8, 598-603).

Olano, C.A. (2007). Plan regional de competitividad de Boyacá 2008-2032. Tunja: Gobernación de Boyacá.

Ordóñez, R. (2013). Proceso agroindustrial de la producción de panela. Revista Tecnura, 17(35), 47-54.

Osorio, G. (2007). Manual técnico: buenas prácticas agrícolas BPA y buenas prácticas de manufactura BPM en la producción de caña y panela. Medellín: CTP Print Ltda.

Palacios, M. (2013). Posibilidades para la redefinición del modelo de negocios. Apuntes del CENES, 32(55), 189-212.

Pelissier, S. (2011). Providing security in energy harvesting sensor networks. The 8th Annual IEEE Consumer Communications and Networking Conference-Security and Content Protection, 452-456.

Rentería, C. (2007). Agenda Interna para la Productividad y la Competitividad. Bogotá:: Departamento Nacional de Planeación.

Rodríguez, S.L. (2011). Diagnóstico Tecnológico, Herramienta para la Planeación de la Ciencia, la Tecnología y la Innovación. Caso: Cotecmar. En: Memorias XIV Congreso Latino-iberoamericano de Gestión Tecnológica ALTEC, 10.

Rosero, E. (2011). Diagnóstico en la producción, transformación y comercialización de panela en cabildos indígenas nasa del norte del Cauca, ante la vigencia y aplicación de la Resolución 779 de 2006. Universidad de San Buenaventura. Disponible en: http://www.panelamonitor.org/media/ docrepo/document/files/diagnostico-en-la-produccion-transformacion-y-comercializacion-de-panela-en-cabildos-indigenas-nasa-del-norte-del-ca. pdf. 
Rozo, J. (2008). Plan de desarrollo departamental de Boyacá 2008-2011. Tunja: Gobernación de Boyacá.

Ruiz, CF. (2012). Plan Estratégico Departamental de Ciencia, Tecnología e Innovación de Boyacá 2022. Tunja: Ediciones Antropos Ltda.

Sánchez, A. (2011). A low cost and high efficient acoustic modem for underwater sensor networks. IEEE Oceanic Engineering Society Conference OCEANS 2011, Spain, DOI: 10.1109/OceansSpain.2011.6003428 .

Secretaría de Fomento Agropecuario (2008). Política sector agropecuario departamento de Boyacá. Tunja: Gobernación de Boyacá.

Torres, L. (2009). Caracterización de Firma Espectral a Partir de Sensores Remotos para el Manejo de Sanidad Vegetal en el Cultivo de Palma de Aceite. Revista Palmas, Vol. 30, No. 3, ISSN: 01212923, 30, 63-79.
Zafar, A. (2011). A review of wireless sensors and networks' applications in agriculture. (Elsevier, Ed.) Computer Standards \& Interfaces, 563-572.

Zhang, H. (2008). Predictions of acidity, soluble solids and firmness of pear using electronic nose technique. (Elsevier, Ed.) Journal of Food Engineering, 86, 370-378.

Zhi, A.E. (2010). Wireless Sensor Networks Powered by Ambient Energy Harvesting: An Empirical Characterization. Education and Research Conference (EDERC), 5.

Zhua, Y. (2011). Applications of wireless sensor network in the agriculture environment monitoring. (Elsevier, Ed.) International Workshop on Automobile, Power and Energy Engineering, 156-163.

\section{(C) $(1) \Theta$}

